\title{
A novel design methodology for low pass filter stage of a voltage source inverter
}

\author{
ANIRBAN DE \\ Department of Atomic Energy, 1/AF, Variable Energy Cyclotron Centre, Bidhan Nagar, Kolkata 700064, India \\ e-mail: ade@vecc.gov.in
}

MS received 28 May 2015; revised 20 November 2015; accepted 17 December 2015

\begin{abstract}
A low pass filter, an invariable constituent of a PWM based voltage source inverter, poses several challenges in its design. With a higher rating of the inverter, the volume as well as the cost of the components increases. With time, the filter design has evolved from the simpler first order filter to higher orders, the most popular being the third order LCL filters. Though the attenuation of high frequency components, offered by the higher order filters, has resulted in improved efficiency, the inherent instability of the higher order filter systems requires complex controls for proper functioning. Keeping this in mind, the paper describes the design methodology of a second order LC filter system based on certain performance indices that reflect the ratings of the components.
\end{abstract}

Keywords. Attenuation; capacitance; inductance; inverters; low-pass filters.

\section{Introduction}

Single phase and three phase voltage source inverters (VSI) are typically the final interface between the power grid and the energy source in several applications. Pulse width modulation (PWM) based VSIs invariably constitute a lowpass filter (LPF) stage for attenuating the higher frequencies arising due to the particular switching schemes.

While a first-order $\mathrm{L}$ filter is a simple solution for the LPF stage but the second-order LC filter and more popularly, the third-order LCL filters are fast gaining momentum as the alternatives. With the increase in the order of the filter, additional control schemes are required for system stability that include methods such as current control strategy of a VSI with an LCL filter [1]; a large signal stabilizer design, based on the circle criterion theory [2] to reject resonant oscillations; a nonlinear controller based on the composite nonlinear feedback control theory [3]; a model predictive direct current control strategy [4] incorporating virtual resistor based active damping; and a twodegree-of-freedom PID active damping method to attenuate the resonance that is caused by the LCL filter based systems [5]. Other filter designs include a 7th order LTCL filter [6] which filters the harmonics efficiently but does not decrease the control difficulties.

One of the basic requirements of higher order filter stems from the fact that a higher rating of the inductors (in case of L filter) and capacitors (in case of LC filters) that comprise the lower order filters are not readily available in the market and are often quite difficult to fabricate. Thus the entire design becomes costly and voluminous as well. Work in this regard includes a design methodology [7] to derive the lower value of the inductor based on inverter output peak current and the capacitor is determined according to the requirement of the resonant frequency $\left(f_{r}\right)$ of the LC filter. Another design perspective was based on the relation among the filter, inverter and the controller assuming the load to be a pure resistor for a worst case scenario [8]. In [9], a design was proposed, based on active damping but this too, like others, assumes a 1:1 isolation transformer only. Further developments have included design based on multiple impact factors [10] but here the unit is transformerless. [11] refers to the importance of the filter design and uses a step-up transformer but does not discuss the design of the filter components. The present work is to supplement these by defining several performance indices to characterize a filter system and utilizes the transformer turns ratio i.e. the ratio of the number of turns in the secondary to that in the primary ( $n)$, advantageously in the design. In this, the transformer leakage inductance is considered to be negligible compared to the other inductances in the circuit. The design attempts to offer a practical choice of the filter components and proposes a novel LC filter topology for a $3-\phi$ VSI system that reduces the volume of the filter and assures a second order attenuation thus eliminating the control overhead required in higher order filters.

The organization of the paper is as follows. The functioning of the VSI system in its targeted application in a superconducting magnetic energy storage (SMES) based 
dynamic voltage restorer (DVR) is briefed in section 2 . Section 3 describes the conventional LC filter topologies and its improvisations, exploiting the transformer's properties. In section 4 the proposed system is explained and the results given and the summary is given in section 5 .

\section{The VSI based system}

The VSI is used at the output stage of a SMES system based DVR system (figure 1), aimed at mitigating voltage sag for balanced resistive load. It may here be pointed out that the paper describes the design methodology to be followed for applications targeted to mitigate voltage sag and is aimed for utilities subjected to voltage sag only. So the rectifier system is only a diode rectifier in the present case. In case of applications demanding voltage swell compensation, a major change in the topology will include replacement of the diode rectifier by a controlled (active) rectifier system as the swell mitigation will result in dumping a good amount of active power across the DC bus of the VSI.

The SMES coil of inductance, $\mathrm{L}$, stores energy $\left(1 / 2 L I^{2}, \mathrm{I}\right.$ being the current flowing through the coil) from the utility mains during healthy mains condition via the DC-DC chopper. During voltage sag at the mains, the same chopper maintains the input voltage to the VSI constant. A potential transformer (PT) based low voltage instrumentation isolates, scales and conditions the mains voltage signals for interfacing a multi-channel ADC that feeds digitized data to a DSP based controller (DSC) core. The heart of the controller is a software phase locked loop (SPLL) $[12,13]$ that locks the input phase and evaluates the amplitude error to generate the gate signals of the full bridge VSI following space vector pulse width modulation (SVPWM) [14-17]. The compensating switching voltages, thus generated, are filtered out by the LPF stage and are added in series, via the

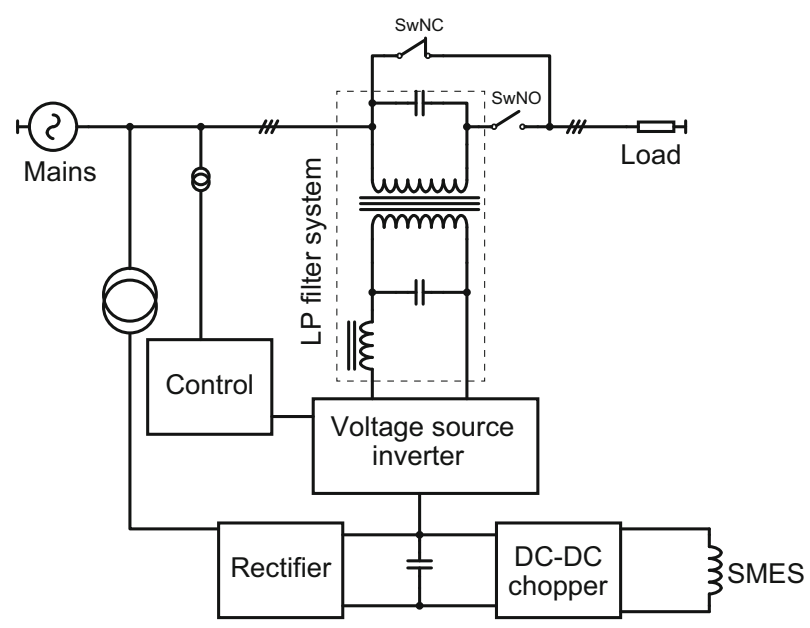

Figure 1. The scheme of the DVR based SMES system. secondary of the $3-\phi \Delta$ /open injection transformer, with the mains voltage $\left(V_{m}\right)$ to keep the load voltage $\left(V_{l}\right)$ constant.

The VSI is designed for a $3-\phi 4$-wire $50 \mathrm{~Hz}$ system feeding a star connected balanced resistive load of rating $50 \Omega\left(R_{l}\right)$ per phase corresponding to a total load of 3.456 KVA and 4.8 A current per phase. As the present discussion considers the design of the filter system only, the input mains are omitted and the three input phases in the model are shorted to the neutral which does not compromise generalization and is equally applicable when the VSI is used as a compensator in the DVR scheme. The gate drivers (Concept make 2SP0115T2Ax-FF450R12ME4) that were selected for the IGBT (Infineon make FF450R12ME4) based full bridge (figure 2) limit the choice of the maximum switching frequency, $19 \mathrm{kHz}$ in this case. As the output fundamental frequency is $50 \mathrm{~Hz}$, the gate triggering is selected two decades (100 times) above that at $5 \mathrm{kHz}$.

In the following discussions, the phase sequences are notated as $a b c$ if $V_{l c}=V_{l b} e^{\frac{2 \pi}{3}}=V_{l a} e^{-\frac{2 \pi}{3}}$ and $a c b$ if $V_{l b}=V_{l c} e^{j \frac{2 \pi}{3}}=V_{l a} e^{-j \frac{2 \pi}{3}}$.

\section{Conventional topologies and their improvisations}

The conventional filter design, for attenuating switching frequency in a VSI, lumps the filter components either in the primary or in the secondary side of the transformer, hereby referred to as $L_{p} C_{p}$ (figure 3 ) and $L_{s} C_{s}$ (figure 4) schemes respectively. In this regard, the component ratings for practical realization of the model are addressed in the following paragraphs.

For the inductor, a higher $\mathrm{AC}$ current flow means a higher power rating requiring an increase in the corresponding bulk of ferrite core that may not be always easily available. This results in stacking of available cores to increase the rating. Due to the brittleness of the core, the pressure during mounting is to be carefully considered as less pressure may cause relative displacement of the cores during energization leading to a change of inductance during operation while higher pressure may cause damage of the material itself. Also, the problem of increased coil dimensions with increasing current may result in inadequate window space as offered by the geometry of the available core. CRGOS laminations could have solved the

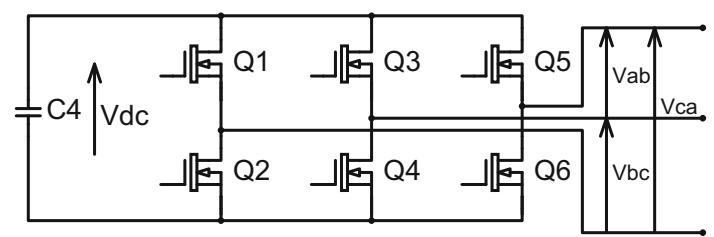

Figure 2. IGBT based full wave VSI system. 


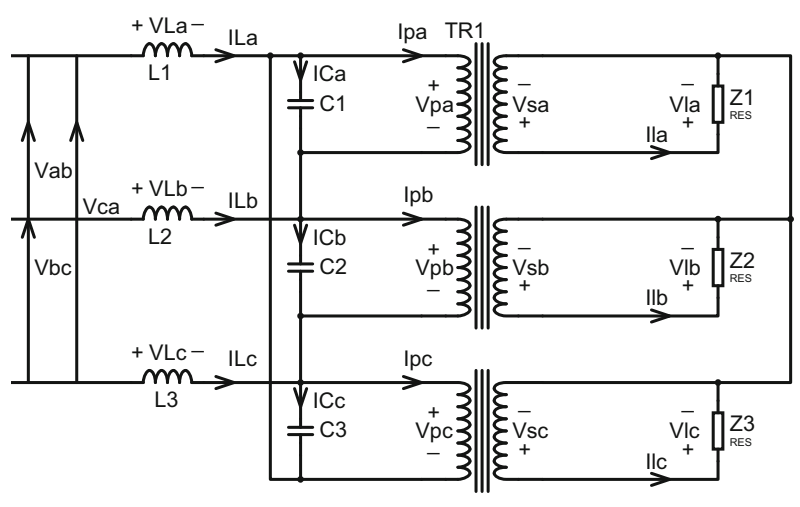

Figure 3. VSI with LC in transformer primary $\left(L_{p} C_{p}\right)$.

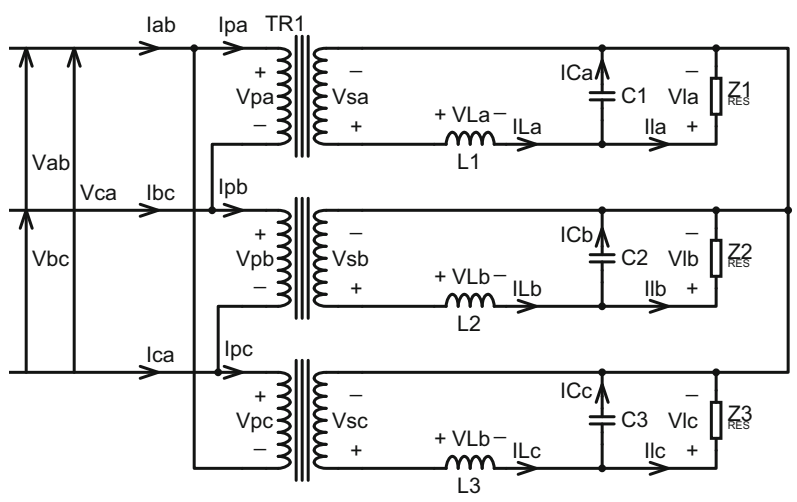

Figure 4. VSI with LC in transformer secondary $\left(L_{s} C_{s}\right)$.

problem but for its poorer frequency response at higher frequencies prevents its use in the present cases.

In the case of the capacitor too, it may be noted that unlike the usual polarized capacitors used for DC power supplies, non-polarized capacitors are required to operate in both the cycles of the input sinusoid. It is also a practical problem of obtaining such a higher value of non-polarized capacitor that has a higher voltage rating and a high ripple current capability. So one of the way-outs is the design of capacitor networks of series-parallel combinations to achieve the required capacitance and the voltage rating. But this will not be a general model and for a change in the requirement, a new network is to be designed to cater to the purpose. This makes the design voluminous as well. Now, in the present case it may be observed that the presence of a transformer, with turns-ratio $(n)>1$, causes the secondary voltage to be stepped up while the secondary current to be stepped down while just the opposite happens if the turnsratio is less than one. This property may be utilized advantageously by placing the $\mathrm{L}$ and $\mathrm{C}$ strategically so that its ratings are favourably affected. As this will finally affect the cut-off and damping ratio, the reverse of the scheme is also tested for completeness. These are illustrated in figures 5 and 6 and referred to as $C_{p} L_{s}$ and $L_{p} C_{s}$ respectively.
In this regard, it may be pointed out that though the $C_{p} L_{s}$ configuration is studied its implementation will cause overcurrent peaks in the IGBTs of the inverter. But even then just for the sake of comparison its filtering performance is discussed in the subsequent subsections.

\subsection{Indices of performance}

From the previous arguments, it may be reasoned that the indices that may categorise a practical filter besides its capability to attenuate the unwanted harmonics are the ratings of the inductor current and the capacitor voltage. Thus the proposed two indicative parameters that will describe the performance of the filter are the capacitor voltage normalized to the load voltage $\left(n_{C l}\right)$ and the inductor current normalized to the load current $\left(n_{L l}\right)$. These, along with the filter transfer function with respect to the inverter line to line voltage $\left(G_{L P}\right)$ are thus formulated by

$$
\left.\begin{array}{rl}
G_{L P}(j \omega) & =\frac{V_{l x}(j \omega)}{V_{x y}(j \omega)} \\
n_{C l}(j \omega) & =\frac{V_{C p x}(j \omega)}{V_{l x}(j \omega)} \text { or, } \frac{V_{C s x}(j \omega)}{V_{l x}(j \omega)} \\
n_{L l}(j \omega) & =\frac{I_{L p x}(j \omega)}{I_{l x}(j \omega)} \text { or, } \frac{I_{L s x}(j \omega)}{I_{l x}(j \omega)}
\end{array}\right\}
$$

where

$$
\begin{aligned}
& x: \text { phase } \mathrm{a}, \mathrm{b} \text { or } \mathrm{c} \\
& y: \text { next phase according to the sequence. }
\end{aligned}
$$

The two formulations, each for $n_{C l}$ and $n_{L l}$, corresponds to whether the capacitor or inductor is placed in the primary of secondary side of the transformer. It may be noted at this stage that further analyses, by decoupling a $3-\phi$ system to three $1-\phi$ systems, will be restricted to phase $a$ only, since in a balanced system, the results are similar for the rest of the phases.

The filter transfer functions and the corresponding indices for the circuits of figures 3, 4, 5, 6 may be listed as in tables 1 and 2. The parameters of the filter transfer functions for different topologies are summarized for comparison in table 3 .

It is thus observed that not only do the magnitudes of $\mathrm{L}$ and $\mathrm{C}$, but $n$ also plays an important role in determining the ratings of the filter components. But unfortunately, the indices and transfer function being coupled to each other, improving one may adversely affect the others. So three sets, each of $n\{0.1,1.0,10\}, \mathrm{L}\{10 \mu \mathrm{H}, 100 \mu \mathrm{H}, 1000 \mu \mathrm{H}\}$ and $\mathrm{C}\{10 \mu \mathrm{F}, 100 \mu \mathrm{F}, 1000 \mu \mathrm{F}\}$ are considered representing three different orders of magnitude to examine the effectiveness of each topology. 


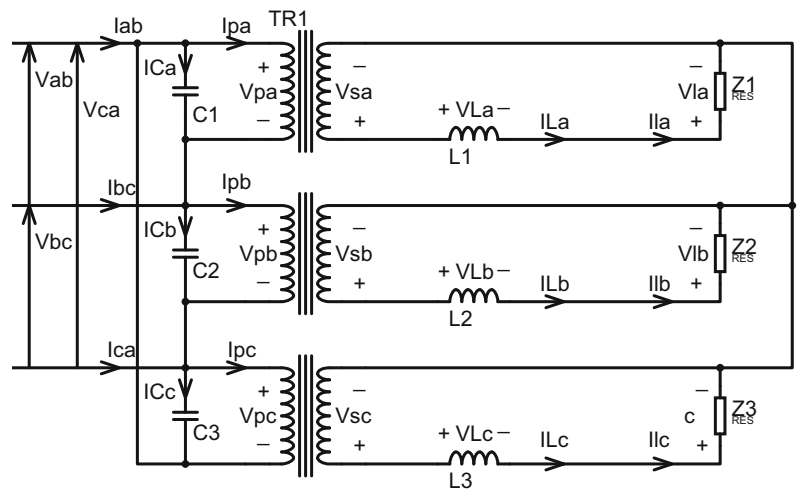

Figure 5. VSI with $\mathrm{C}$ in transformer primary and $\mathrm{L}$ in secondary $\left(C_{p} L_{s}\right)$.

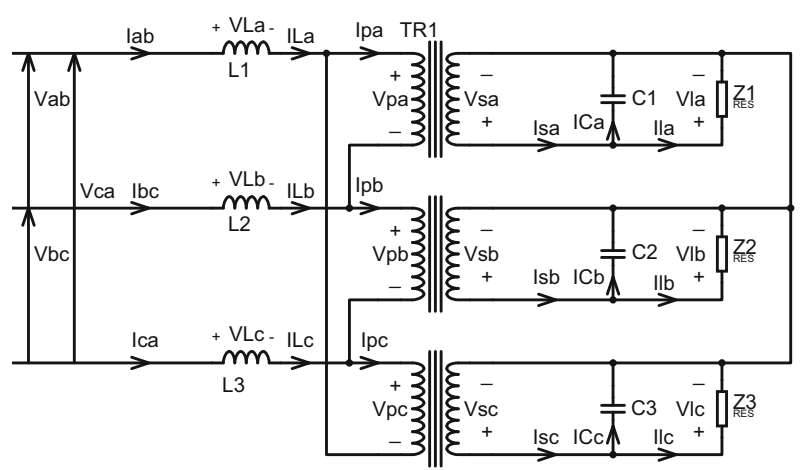

Figure 6. VSI with $\mathrm{L}$ in transformer primary and $\mathrm{C}$ in secondary $\left(L_{p} C_{s}\right)$.

Table 1. Load voltage normalized to VSI output line voltage.

\begin{tabular}{lcc}
\hline Figure & Topology & $G_{L P}(j \omega)$ \\
\hline Figure 3 & LpCp & $\frac{n /\left(3 L_{p} C_{p}\right)}{\left(-\omega^{2}+j \frac{n^{2}}{R_{l} C_{p}} \omega+\frac{1}{3 L_{p} C_{p}}\right)}$ \\
Figure 4 & LsCs & $\frac{n /\left(L_{s} C_{s}\right)}{\left(-\omega^{2}+j \frac{1}{R_{l} C_{s}} \omega+\frac{1}{L_{s} C_{s}}\right)}$ \\
Figure 5 & CpLs & $\frac{n R_{l} / L_{s}}{\left(\frac{R_{l}}{L_{s}}+j \omega\right)}$ \\
Figure 6 & LpCs & $1 /\left(3 n L_{p} C_{p}\right)$ \\
\hline
\end{tabular}

It may here be noted that as the $C_{p} L_{s}$ configuration is a first order system, it will provide attenuation, poorer than the other three. So from this perspective too, the other being its configuration adversely affecting the IGBT functioning, the configuration is omitted from further discussions.

\subsection{Selecting the condition to compare the performance}

As $n_{C l}$ and $n_{L l}$ are also functions of frequency, we select a common frequency viz., the desired system (load) frequency i.e. $50 \mathrm{~Hz}$, for comparing the parameters. Now, to choose the filter cut-off frequency we may follow [7] which proposed the criteria $10 f_{s} \leq f_{r}=\frac{1}{2 \pi \sqrt{L C}} \leq M f_{s w}\left(f_{s}, f_{s w}\right.$ are respectively the system frequency and the PWM switching frequency) with $M$ in the range [0.1, 0.5]. But considering the present case with system frequency of $50 \mathrm{~Hz}$ and PWM switching frequency of $5 \mathrm{kHz}$, the lower limit $\left(10 f_{s}=500 \mathrm{~Hz}\right)$ coincides with the lower range $\left(0.1 f_{s w}\right)$ of the upper limit. This happens as the difference of order between the system frequency and the switching frequency is not very high. For this reason, an additional constraint is included in the design viz., the settling time for the filter.

As the target design is a DVR system that is supposed to mitigate voltage sag at the mains within two cycles (due to the presence of the electrical contactor that has the highest response time among the other components in the circuit) with a voltage tolerance of $10 \%$, it is expected that the filter settling time is much less than that. So the settling time $\left(t_{\text {settle }}\right)$ is taken to be a quarter of a $50 \mathrm{~Hz}$ cycle, i.e., $5 \mathrm{~ms}$ and a $5 \%$ settling band is considered. From definition, this will correspond to

$$
\zeta \omega_{n}=-\frac{\ln 0.05}{t_{\text {settle }}}=559.15 \mathrm{rad} / \mathrm{s}
$$

With this, the damping constant and the attenuation at $f_{s w}$ with respect to $f_{s}$ for various $f_{n}$ is tabulated in table 4 . From this table it is observed that if $3 f_{s} \leq f_{n} \leq 5 f_{s}$, then the attenuation is more than $50 \mathrm{~dB}$ as well as the circuit is not very much underdamped. On this basis, the cut-off frequency is selected at five times the system frequency.

\subsection{Comparing the ratings of $L$ and $C$ based on the fixed cut-off frequency}

From table 3 it may be observed that the inductance will vary inversely as the capacitance and vice versa but the proportionality constant will depend on the particular topology. Thus, for a constant cut-off frequency and capacitance, the $L_{p} C_{p}$ configuration, though always gives a lower inductance (capacitance) than the $L_{s} C_{s}$ ( $\sqrt{3}$ times less) but it is higher (by a factor of $n$ ) than what is offered by $L_{p} C_{s}$ if the transformer used is a step-up one.

The current rating of the inductor at the power frequency is reflected by the magnitude of $n_{L l}$. Its variation as a function of $n$ and capacitor value for each of the three topologies, viz., $L_{p} C_{p}, L_{s} C_{s}$ and $L_{p} C_{s}$, is shown in figure 7. The family of graphs for each topology corresponds to the variation of $n_{L l}$ vs $n$ for different capacitor values. Figure 8 
Table 2. $n_{L l}(j \omega)$ and $n_{C l}(j \omega)$.

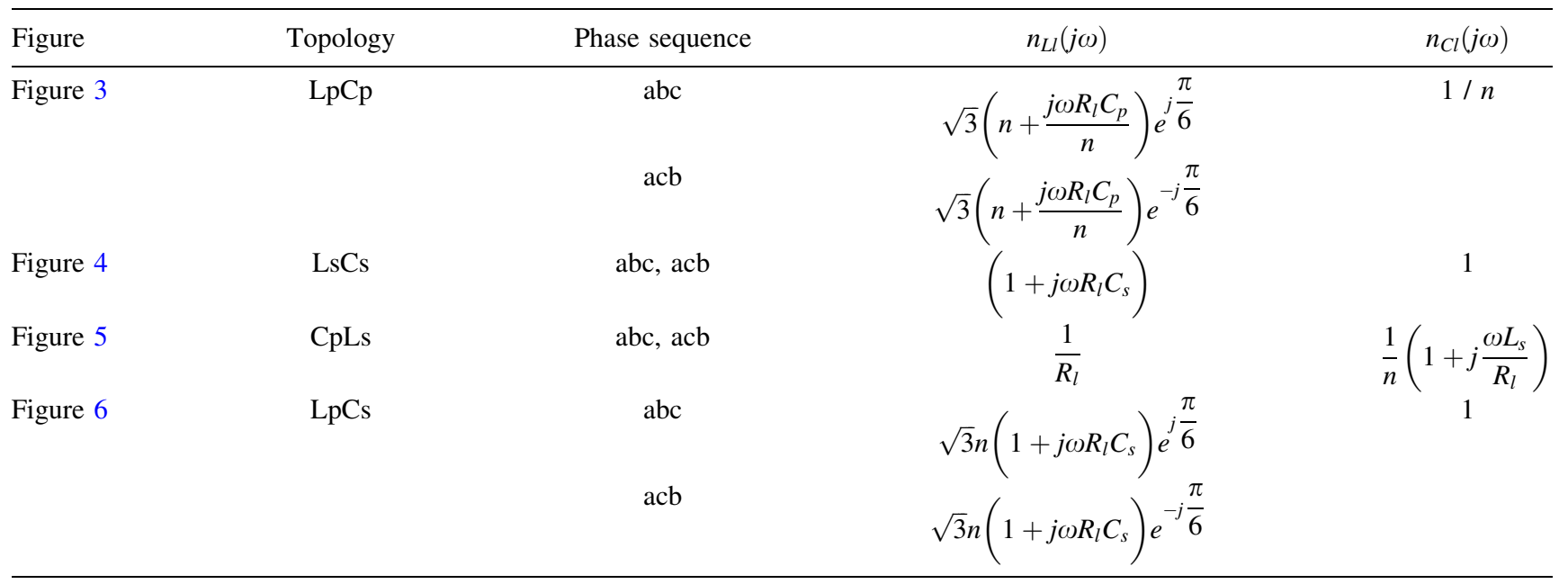

Table 3. Comparison of parameters of the filter transfer functions for various LPF topologies.

\begin{tabular}{lccc}
\hline Topology & $\omega_{n}$ & $\zeta$ & $\mathrm{k}$ \\
\hline LpCp & $\frac{1}{\sqrt{3 L_{p} C_{p}}}$ & $\frac{\sqrt{3} n^{2}}{2 R_{l}} \sqrt{\frac{L_{p}}{C_{p}}}$ & $n$ \\
LsCs & $\frac{1}{\sqrt{L_{s} C_{s}}}$ & $\frac{1}{2 R_{l}} \sqrt{\frac{L_{s}}{C_{s}}}$ & $n$ \\
CpLs & $\frac{R_{l}}{L_{s}}$ & - & $n$ \\
LpCs & $\frac{1}{n \sqrt{3 L_{p} C_{s}}}$ & $\frac{\sqrt{3} n}{2 R_{l}} \sqrt{\frac{L_{p}}{C_{s}}}$ & $n$ \\
\hline
\end{tabular}

Table 4. Comparison of attenuation and damping constant for various cut-off frequencies.

\begin{tabular}{lcc}
\hline & $20 \log \left|\frac{G_{L P}\left(j f_{s w}\right)}{G_{L P}\left(j f_{s}\right)}\right|$ & $\zeta$ \\
\hline $50 \mathrm{~Hz}$ & $68.38 \mathrm{~dB}$ & 1.91 \\
$100 \mathrm{~Hz}$ & $66.28 \mathrm{~dB}$ & 0.95 \\
$150 \mathrm{~Hz}$ & $61.05 \mathrm{~dB}$ & 0.64 \\
$200 \mathrm{~Hz}$ & $56.20 \mathrm{~dB}$ & 0.48 \\
$250 \mathrm{~Hz}$ & $52.27 \mathrm{~dB}$ & 0.38 \\
$300 \mathrm{~Hz}$ & $49.04 \mathrm{~dB}$ & 0.32 \\
$350 \mathrm{~Hz}$ & $46.31 \mathrm{~dB}$ & 0.27 \\
$400 \mathrm{~Hz}$ & $43.95 \mathrm{~dB}$ & 0.24 \\
$450 \mathrm{~Hz}$ & $41.86 \mathrm{~dB}$ & 0.21 \\
$500 \mathrm{~Hz}$ & $40.00 \mathrm{~dB}$ & 0.19 \\
\hline
\end{tabular}

shows the variation of $n_{C l}$ with $n$ for each of the topologies that reflect similarly the voltage rating of the filter capacitors. From these, the following points are of significance for the present purpose.

(1) For the same cut-off frequency and the capacitance (inductance) value, the inductance (capacitance) required is always lower in $L_{p} C_{p}$ than in $L_{s} C_{s}$ but for a step-up transformer, the lowest inductance (capacitance) is offered by the $L_{p} C_{s}$ topology.

(2) The current rating of the inductor in $L_{S} C_{s}$ is the lowest for the complete range of the turns-ratio and the load considered, while that in the $L_{p} C_{p}$ becomes more or less same for large $n(>10)$ that also matches the $L_{p} C_{s}$ plot if $C_{s}$ is of the order of $10 \mu \mathrm{F}$.

(3) The voltage rating of the capacitors used in $L_{s} C_{s}$ and $L_{p} C_{s}$ are same and constant which is higher/equal/lower than that for the $L_{p} C_{p}$ situation for a step-up/isolation/ step-down transformer respectively.

\subsection{Summarizing the observations}

From the previous two subsections it is observed that for lower ratings (voltage, current, inductance and capacitance values) of the filter component is favoured if the transformer selected is a step-up one. It is also seen that with a step-up transformer, the inductance (capacitance) requirement is the lowest for the $L_{p} C_{s}$ configuration for identical value of the capacitance (inductance). Thus the $L_{p} C_{s}$ configuration would have been the best option but for the fact that the voltage rating of the capacitor in this case, or more generally in the case when it is placed in the secondary side, becomes same as the load voltage. The availability of such capacitors is poor and they are also very costly. To counter this limitation, a new topology is proposed and discussed in the next section.

\section{Proposed filter configuration}

Considering the previous rationale, a step-up transformer with $\mathrm{C}$ distributed in both the primary and secondary $\left(L_{p} C_{p} C_{s}\right)$ is proposed (figure 9$)$ so that a better filtering is achieved with reduced volume of the filter. 

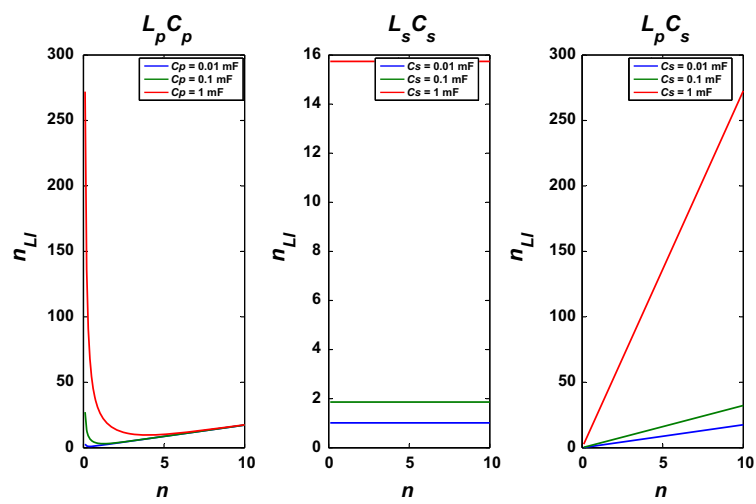

Figure 7. $n_{L l}$ vs $n$ for different capacitor values for the configurations (from left to right) $L_{p} C_{p}, L_{s} C_{s}$ and $L_{p} C_{s}$.

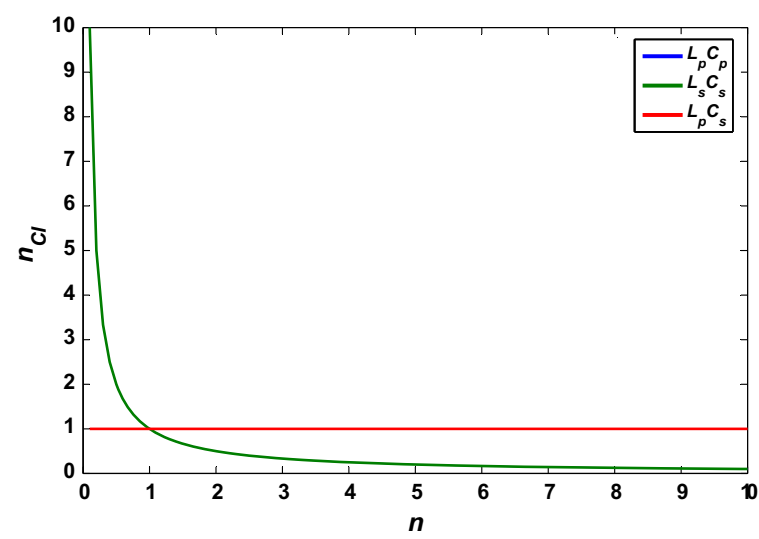

Figure 8. $n_{C l}$ vs $n$ for configurations $L_{p} C_{p}, L_{s} C_{s}$ and $L_{p} C_{s}$.

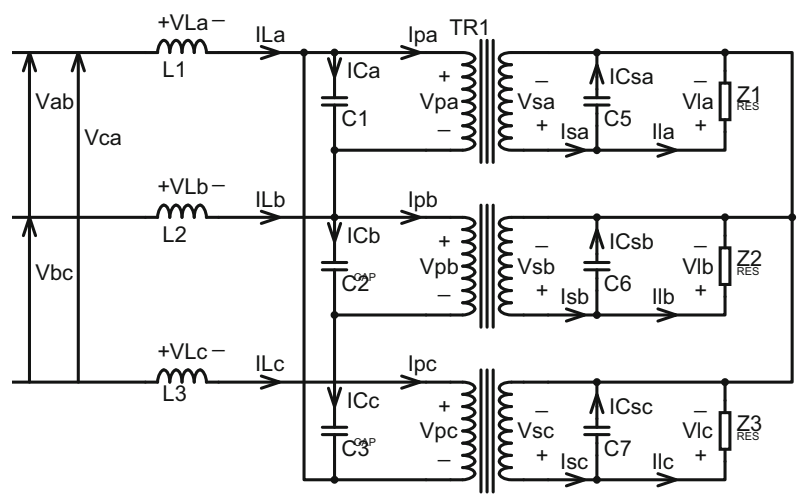

Figure 9. VSI with $\mathrm{L}$ in transformer primary and $\mathrm{C}$ distributed in both sides of transformer $\left(L_{p} C_{p} C_{s}\right)$.

\subsection{Transfer function and performance indices}

Analysing the topology, the indices (subscripts indicating phase sequences) are listed as

$$
\begin{aligned}
n_{L l}(j \omega)_{a b c} & =\sqrt{3}\left(n+j \omega R_{l}\left(\frac{C_{p}}{n}+n C_{s}\right)\right) e^{j \frac{\pi}{6}} \\
n_{L l}(j \omega)_{a c b} & =\sqrt{3}\left(n+j \omega R_{l}\left(\frac{C_{p}}{n}+n C_{s}\right)\right) e^{-j \frac{\pi}{6}} \\
n_{C p l}(j \omega) & =1 / n \\
n_{C s l}(j \omega) & =1 \\
G_{L P}(j \omega) & =\frac{\frac{n}{3 L_{p}\left(C_{p}+n^{2} C_{s}\right)}}{\left(-\omega^{2}+j \frac{n^{2}}{R_{l}\left(C_{p}+n^{2} C_{s}\right)} \omega+\frac{1}{3 L_{p}\left(C_{p}+n^{2} C_{s}\right)}\right)}
\end{aligned}
$$

which gives

$$
\begin{aligned}
\omega_{n} & =\frac{1}{\sqrt{3 L_{p}\left(C_{p}+n^{2} C_{s}\right)}} \\
\zeta & =\frac{\sqrt{3} n^{2}}{2 R_{l}} \sqrt{\frac{L_{p}}{\left(C_{p}+n^{2} C_{s}\right)}} \\
k & =n .
\end{aligned}
$$

Choosing the same sets of $C_{p}$ and $C_{s}$ and considering a 10:1 transformer, the current rating of the inductor normalized to the load current for various values of $C_{p}$ and $C_{s}$ is shown in figure 10. In the plot, $n_{L l}$ is plotted vs $C_{p}$ for different values of $C_{s}$. It is thus obvious that for a given secondary capacitance, the increase in primary capacitance does not appreciably affect the inductor current rating. But due to the scarcity and cost of the secondary capacitor with a high voltage rating, its value cannot be arbitrarily increased. Thus the primary capacitor, with a voltage rating one-tenth as compared to the secondary capacitor, may be very well used to fine tune the requirement of the cut-off frequency without a significant change of the inductor current rating.

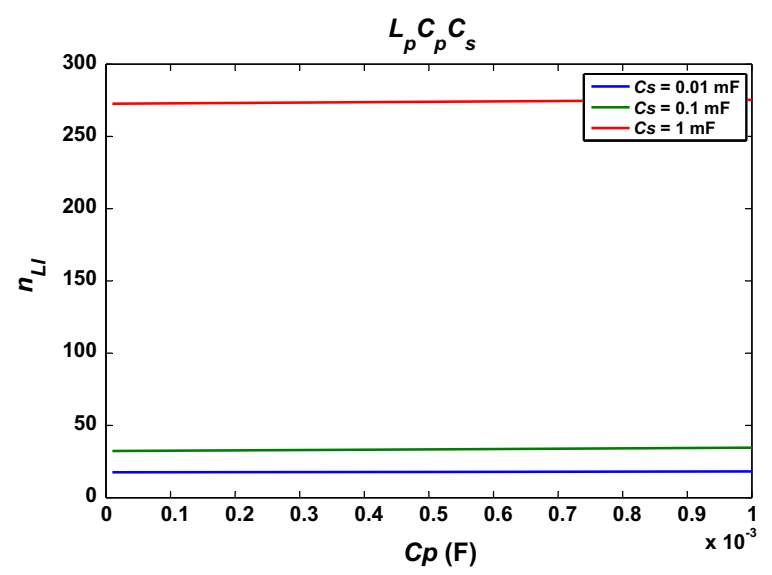

Figure 10. $n_{L l}$ vs $C_{p}$ for different $C_{s}$ for a 10:1 transformer in $L_{p} C_{p} C_{s}$ configuration. 

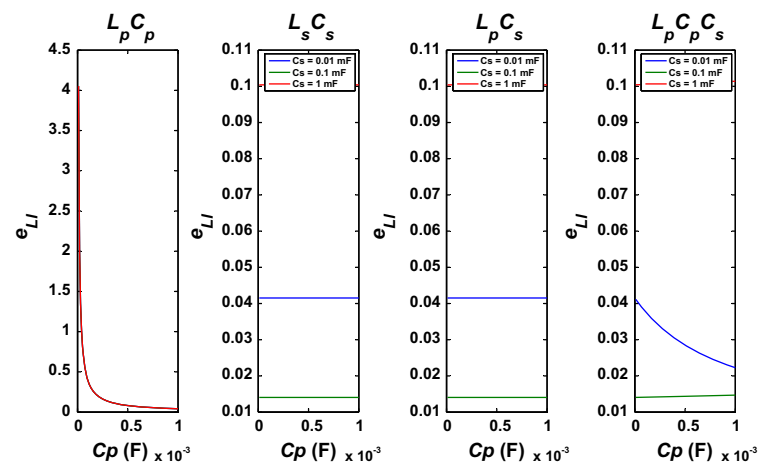

Figure 11. $e_{L l}$ vs $C_{p}$ with different $C_{s}$ for various filter configurations.

\subsection{Defining energy function based performance indices}

At this stage, an additional performance index is defined for the inductor based on the stored energy requirement of the filter devices. Thus defining

$$
\begin{gathered}
e_{L l}=L_{p} n_{L l}(j \omega)^{2} \text { or } L_{p} n_{L l}(j \omega)^{2} \text { depending on which side } \\
\text { the inductor is placed }
\end{gathered}
$$

it may be inferred that the figure will reflect both the inductance as well as the current it will be carrying and thus higher the value of the product $\left(e_{L l}\right)$, higher will be the cost of the inductor. This is obvious as more is the energy stored, greater will be the required volume of the filter and thus greater will be its cost.

So, considering the same cut-off frequency and a constant turns-ratio, the plot of the energy function for various values of the filter capacitors is shown in figure 11 from which it may be concluded that if $10 \mu \mathrm{F}<C_{s}<100 \mu \mathrm{F}$, the energy function is lowest in the case of $L_{p} C_{p} C_{s}$.

\subsection{Implementation of the model}

From figure 10 it is seen that the lowest $n_{L l}$ corresponds to $C_{s}=10 \mu \mathrm{F}$ range i.e., the lowest of the range that was considered and figure 11 dictates $10 \mu \mathrm{F}<C_{s}<100 \mu \mathrm{F}$ for lower energy function for the inductor. Additionally, the plot shows that if $C_{s}$ is in the $10 \mu \mathrm{F}$ range, $e_{L l}$ is a decreasing function of $C_{p}$.

Considering these, the model was implemented with $C_{s}=14.7 \mu \mathrm{F}$ (corresponding to a still lower value of $e_{L l}$ than $10 \mu \mathrm{F}$ ) and $C_{p}=360.0 \mu \mathrm{F}$. With the conditions previously stated, the inductor values come as $L_{p}=73.82 \mu \mathrm{H}$. The implemented inductance had a final value of $80.0 \mu \mathrm{H}$. The designed transformer too deviated from the design condition and the final turns-ratio was measured as 11.53. The Bode plot of the implemented filter is shown in

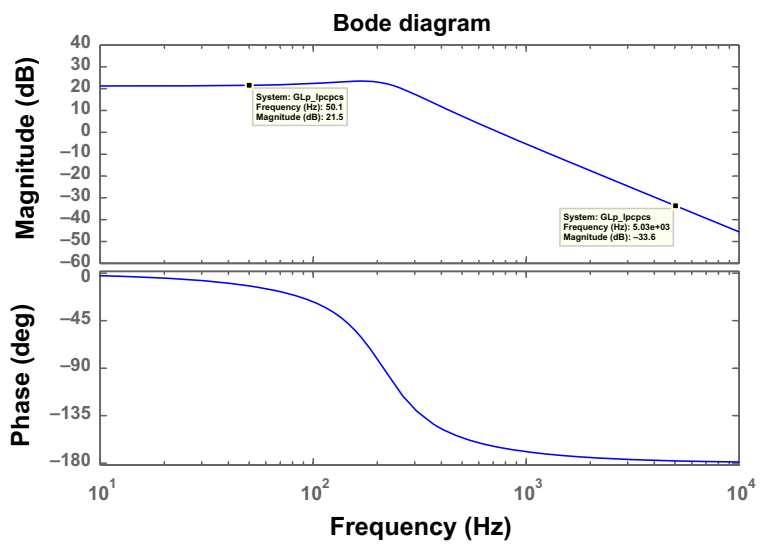

Figure 12. Bode plot for the implemented $L_{p} C_{p} C_{s}$.

figure 12 which shows an attenuation at $5 \mathrm{kHz}$ with respect to $50 \mathrm{~Hz}$ is coming as

$$
\text { attenuation, } \begin{aligned}
\Delta M & =\mathrm{dB} \text { near } 50 \mathrm{~Hz}-\mathrm{dB} \text { near } 5 \mathrm{kHz} \\
& \sim 21.5 \mathrm{~dB}-(-33.6) \mathrm{dB} \\
& =55.1 \mathrm{~dB}
\end{aligned}
$$

The cut-off frequency of the filter calculated according to (5) gives the value of $213.56 \mathrm{~Hz}$.

The load voltage as obtained from the storage oscilloscope data is plotted in figure 13 and the calculated power spectrum is plotted in figure 14 .

The system was also run as the DVR and the data obtained is plotted in figure 15. It is observed that though the gate is operating almost instantaneously as the voltage sag occurs, the load takes around two cycles to be fully compensated. This is due to the delayed response of the electrical contacts that take around two cycles to be completely closed. The response was similar when the mains voltage was restored to the normal value.

\subsection{Discussions}

(1) With the utilization of the new topology, targetted to reduce the ratings of the filter components, the filtering itself was not compromised and the attenuation at the switching frequency with respect to the system frequency is $\sim 105 \mathrm{~dB}-50 \mathrm{~dB}=55 \mathrm{~dB}$ matches the design (7) and is more than $40 \mathrm{~dB}$, the standard attenuation requirement from a second-order filter. This also justifies the choice of the cut-off frequency. Thus without the use of a higher order filter, the attenuation offered had been sufficient.

(2) Due to the inherent stability of a second order filter system, there is no extra control system requirement as required for a filter system of order three or more. 


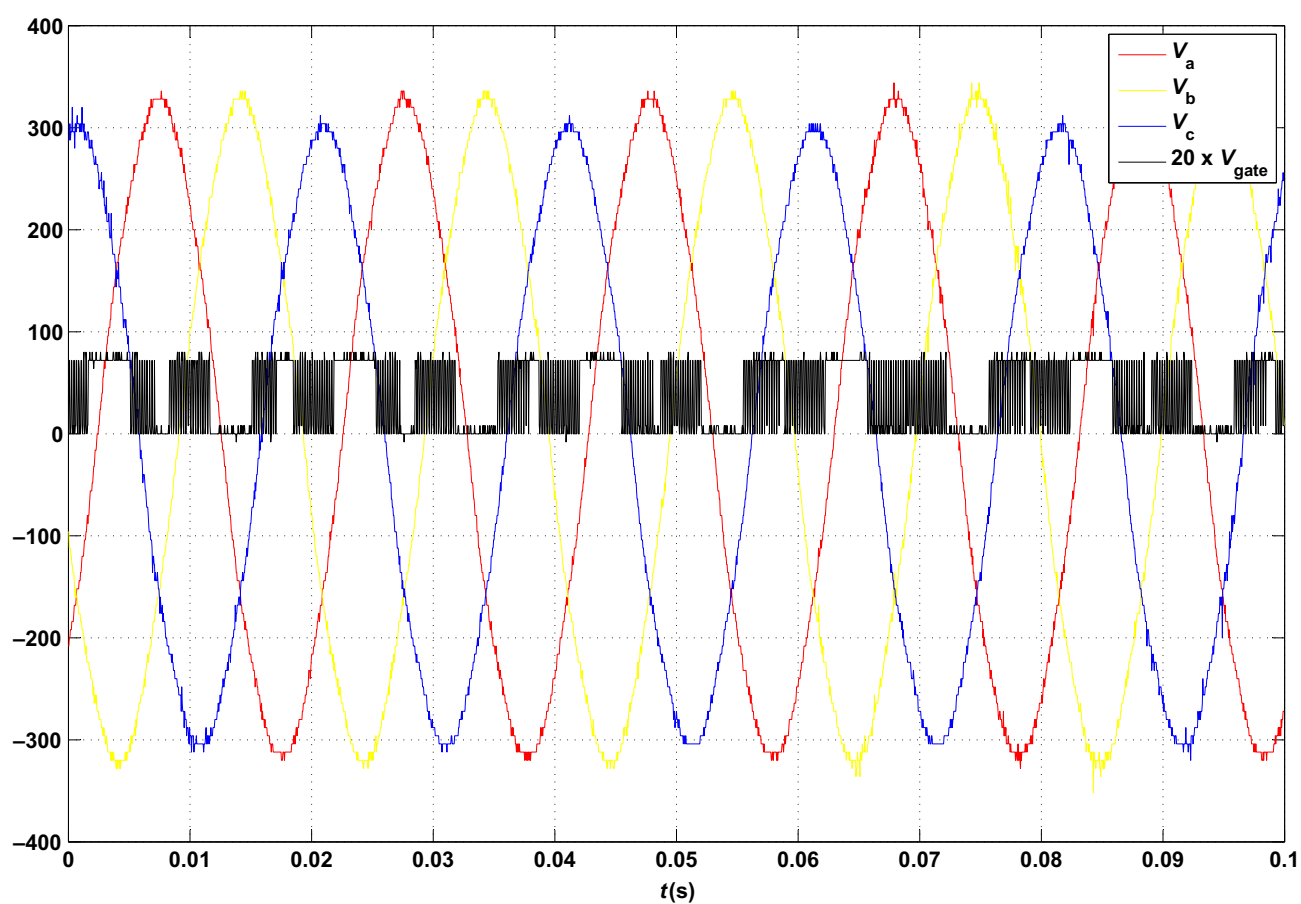

Figure 13. Readings of the $3-\phi$ load voltages along with one of the switching sequences (amplified 20 times).
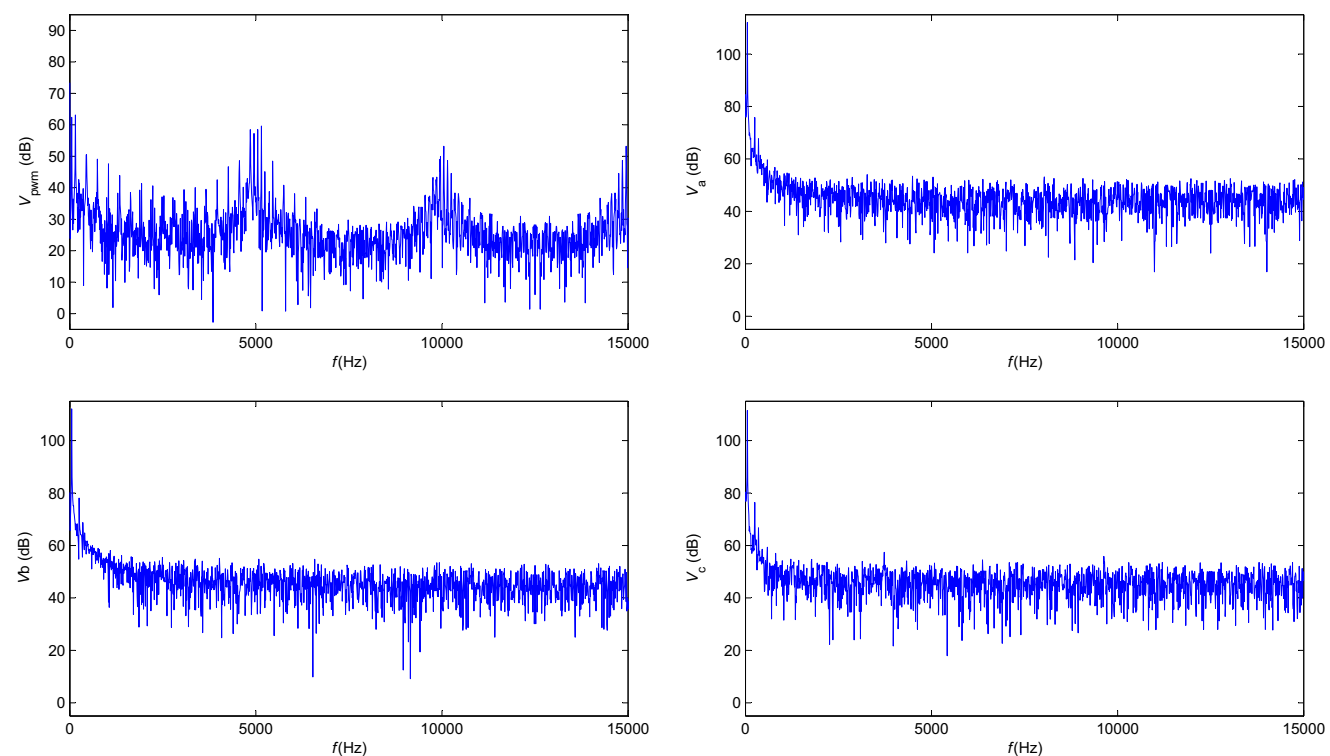

Figure 14. Power spectrum of the $3-\phi$ output waveforms and the PWM gate drive (top left) with the $L_{p} C_{p} C_{s}$ topology.

(3) An added spin-off for using a step-up transformer is its effect in reducing the rating of the DC bus voltage at the input of the VSI. This will allow lesser insulation at the bus and the capacitor, and reduces the voltage rating of the corresponding power electronic switches which may be favourable from their operational point of view.

(4) The system was applied in the DVR assembly and there too the performance was according to the requirement. 

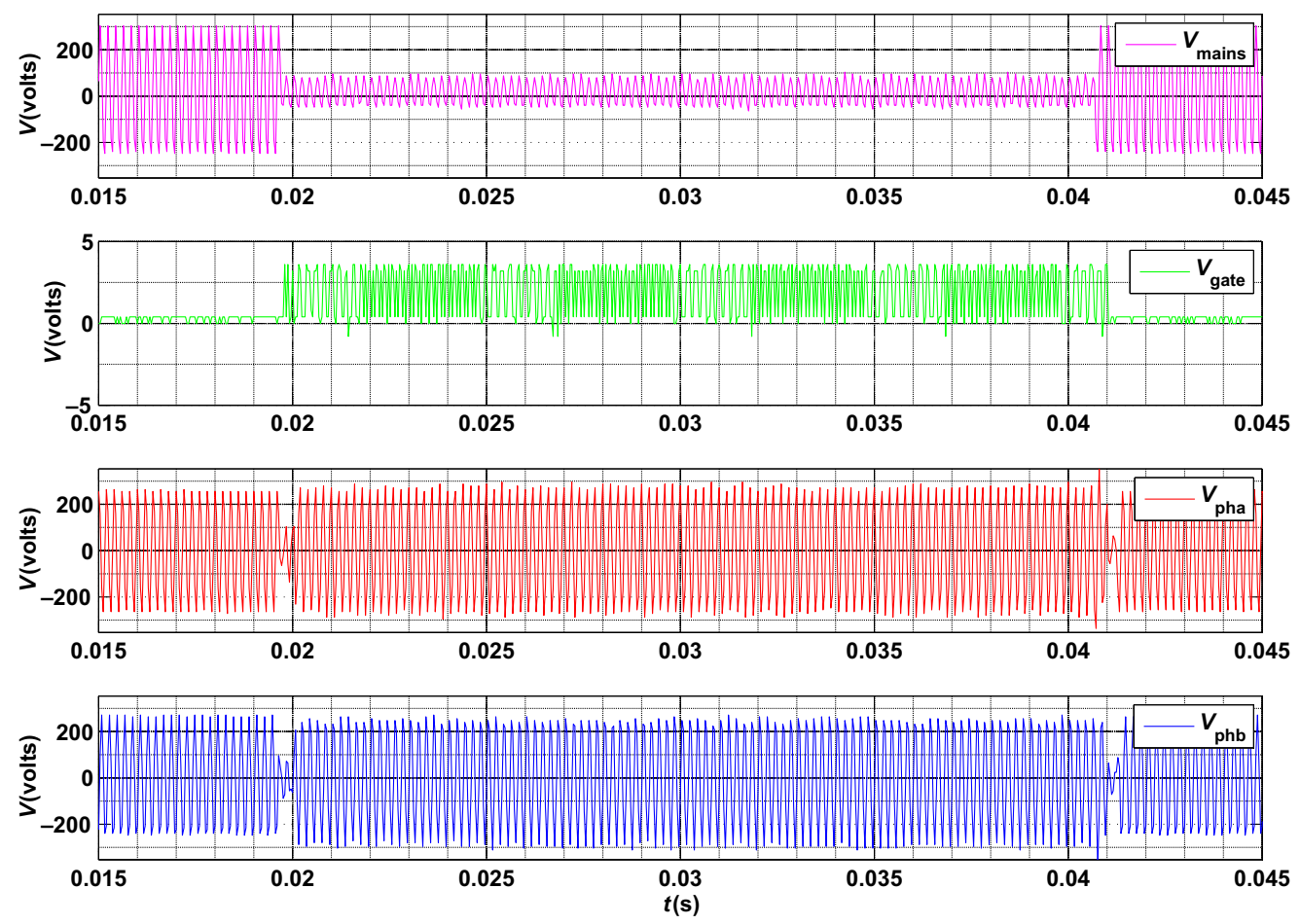

Figure 15. Plots showing the voltage sag and restoration at the mains (top), the gate driver response and output voltages of two of the load phases (bottom two).

\section{Conclusion}

The paper has proposed a novel technology for reducing the ratings of the filter components of a VSI based on a set of performance indices. In the process, the attenuation of more than $40 \mathrm{~dB}$ has been achieved at the switching frequency. The implemented system further corroborates the design values. The inherent stability of a second-order system justifies no requirement of extra control system due to the inclusion of the filter. The VSI was also employed to be used as voltage injector in a DVR and the performance was as per requirement.

\section{Acknowledgements}

The author acknowledges the project members of SMES Technology Development and the colleagues of Power Electronics and Magnet Coil Development Division, VECC who wholeheartedly supported the research and developmental works. The author wishes to acknowledge the associate editor and the anonymous reviewer for valuable comments and suggestions.

\section{Appendix: Notations}

- Mains voltage: $V_{m}=\left[\begin{array}{lll}V_{m a} & V_{m b} & V_{m c}\end{array}\right]^{T}$
- Load

- Voltage: $V_{l}=\left[\begin{array}{lll}V_{l a} & V_{l b} & V_{l c}\end{array}\right]^{T}$

- Current: $I_{l}=\left[\begin{array}{lll}I_{l a} & I_{l b} & I_{l c}\end{array}\right]^{T}$

- Power transformer

- Secondary voltage: $V_{s}=\left[\begin{array}{lll}V_{s a} & V_{s b} & V_{s c}\end{array}\right]^{T}$

- Secondary current: $I_{s}=\left[\begin{array}{lll}I_{s a} & I_{s b} & I_{s c}\end{array}\right]^{T}$

- Primary voltage: $V_{p}=\left[\begin{array}{lll}V_{p a} & V_{p b} & V_{p c}\end{array}\right]^{T}$

- Primary current: $I_{p}=\left[\begin{array}{lll}I_{p a} & I_{p b} & I_{p c}\end{array}\right]^{T}$

- Inductor

- Inductance when placed in transformer primary: $L_{p}$

- Inductance when placed in transformer secondary: $L_{s}$

- Current: $\quad I_{L w}=\left[\begin{array}{lll}I_{L w a} & I_{L w b} & I_{L w c}\end{array}\right]^{T}, \quad w:$ primary $(p)$ or secondary $(s)$ winding

- Voltage: $V_{L w}=\left[\begin{array}{lll}V_{L w a} & V_{L w b} & V_{L w c}\end{array}\right]^{T}$

- Capacitor placed in transformer primary

- Capacitance: $C_{p}$

- Voltage: $V_{C p}=\left[\begin{array}{lll}V_{C p a} & V_{C p b} & V_{C p c}\end{array}\right]^{T}$

- Current: $I_{C p}=\left[\begin{array}{lll}I_{C p a} & I_{C p b} & I_{C p c}\end{array}\right]^{T}$

- Capacitor placed in transformer secondary

- Capacitance: $C_{s}$

- Voltage: $V_{C s}=\left[\begin{array}{lll}V_{C s a} & V_{C s b} & V_{C s c}\end{array}\right]^{T}$

- Current: $I_{C s}=\left[\begin{array}{lll}I_{C s a} & I_{C s b} & I_{C s c}\end{array}\right]^{T}$ 


\section{References}

[1] Papavasiliou A, Papathanassiou S, Manias S and Demetriadis G 2007 Current control of a voltage source inverter connected to the grid via LCL filter. In: Proceedings of IEEE Power Electronics Specialists Conference 2007, pages 2379-2384

[2] Houari A, Renaudineau H, Nahid-Mobarakeh B, Martin J-P, Pierfederici S and Meibody-Tabar F 2014 Large signal stability analysis and stabilization of converters connected to grid through LCL filters. IEEE Trans. Ind. Electron. 61: 6507-6516.

[3] Eren S, Pahlevaninezhad M, Bakhshai A and Jain P, 2013 Composite nonlinear feedback control and stability analysis of a grid-connected voltage source inverter with LCL filter. IEEE Trans. Ind. Electron. 60: 5059-5074

[4] Scoltock J, Geyer T and Madawala U 2013 Model predictive direct current control for a grid-connected converter: LCLfilter versus 1-filter. In: Proceedings of IEEE International Conference on Industrial Technology 2013, pages 576-581.

[5] Hanif M, Khadkikar V, Xiao W and Kirtley J 2014 Two degrees of freedom active damping technique for LCL filterbased grid connected pv systems. IEEE Trans. Ind. Electron. 61: 2795-2803.

[6] Xu J, Yang J, Ye J, Zhang Z and Shen A 2014 An LTCL filter for three-phase grid-connected converters. IEEE Trans. Power Electron. 29: 4322-4338

[7] Zhang H, Yao S, Zhu Z, Cao J and Mao T 2014 Design, development and testing of a DSP based dynamic voltage restorer. In: 6th International Conference on Electronics and Information Engineering, pp. 133-141

[8] Kim H, Kim J-H and Sul S-K 2004 A design consideration of output filters for dynamic voltage restorers. In: Proceedings of IEEE 35th Annual Power Electronics Specialists Conference 2004, vol. 6, pp. 4268-4272

[9] Guo S and Liu D 2011 Analysis and design of output LC filter system for dynamic voltage restorer. In: Proceedings
IEEE 26th Applied Power Electronics Conference and Exposition 2011, pages 1599-1605

[10] Chen G, Zhu M and Xu C 2014 Parameter optimization of the LC filters based on multiple impact factors for cascaded H-bridge dynamic voltage restorers. J. Power Electron. 14: 165-174

[11] El-Shennawy T, Moussa A, El-Gammal M and AbouGhazala A 2010 A dynamic voltage restorer for voltage sag mitigation in a refinery with induction motors loads. Am. J. Eng. Appl. Sci. 3: 144-151

[12] Ramachandaramurthy V, Fitzer C, Arulampalam A, Zhan C, Barnes M and Jenkins N 2002 Control of a battery supported dynamic voltage restorer. In: Proceedings of IEE Generation, Transmission and Distribution, 149: 533-542

[13] Zhan C, Ramachandaramurthy V K, Arulampalam A, Fitzer C, Kromlidis S, Barnes M and Jenkins N 2001 Dynamic voltage restorer based on voltage space vector PWM control. In: Proceedings of IEEE 16th Applied Power Electronics Conference and Exposition 2001, vol. 2, pp. 1301-1307

[14] Gajdůšek I 2012 Programable laboratory invertor and space vector PWM. URL http://www.feec.vutbr.cz/EEICT/2005/ sbornik/03-Doktorske_projekty/04-Silnoprouda_elektrotechn ika_a_elektroenergetika/03-SmileCz. accessed Jun 30, 2012

[15] i Felix J P 2002 Modulation and control of three-phase PWM multilevel converters. $\mathrm{PhD}$ thesis, Technical University of Catalonia, Spain

[16] Mohan N, Sulkowski W, Jose P and Brekken T 2012 Including voltage space vector PWM in undergraduate courses. URL http://et.upt.ro/admin/tmpfile/fileE1224952113 file490349319450a. accessed Jul 09, 2012.

[17] Wang Z G, Jin J X, Guo Y G, Zhu J G 2008 Svpwm techniques and applications in hts pmsm machines control. $J$. Electron. Sci. Technol. China 6: 191-197. 